\title{
Innovative Approach of EOQ Structure for Decaying Items with Time Sensitive Demand, Cash- Discount, Shortages and Permissible Delay in Payments
}

\author{
R. P. Tripathi ${ }^{1}$
}

Accepted: 21 March 2021 / Published online: 26 April 2021

(c) The Author(s), under exclusive licence to Springer Nature India Private Limited 2021

\begin{abstract}
Growing business process and rising aggressive conditions are encouraged to use the inventory control scheme and components in an ideal way. Cash discount and permissible delay are beneficial for vendor and buyer both. This study considers an EOQ model through demand rate depends on the time. A lower or higher time leads to lower or higher demand after feedback vice versa. In this paper deterioration, cash- discount, shortages and permissible delay are also considered. Mathematical models are discussed under four different states of affair. Solution method is given for finding the finest answer. The main aim is to maximize total profit. Numerical examples are provided for all four dissimilar situations. Optimal values with strictures are calculated to analyze the sensitivity investigation of optimal strategy concerning the parameters of the system. It is revealed that the total income is concave by means of cycle time.
\end{abstract}

Keywords Cash- discount; Inventory; Deterioration · Shortages · Demand · Trade credit

Mathematics Subject Classification 90B05

\section{Introduction}

The production of commodities is the first stage of manufacture. Three stage system, a manufacturer, seller and market are considered in the process. The retailer's term represents for part of the system works as a bridge between market and manufacturer. EOQ models begin by taking into account that the demand rate was stable along with cycle time. This assumption was a serious restriction because in real life, demand of a commodity can depend on such multiple issue at the time, stock - level, quantity, selling price, lead time, advertisings, rebate, exponential etc.

Silver and Peterson [1] pointed out that the demand rate of some goods may be influenced by the stock stage. Indeed, huge heaps of supplies exhibited in a superstore sometimes guides

$\triangle$ R. P. Tripathi

tripathi_rp0231@rediffmail.com

1 Department of Applied Sciences and Humanities, KNIT, Sultanpur, Uttar Pradesh, India 
buyers to purchase more. There are two common rules for the inventory manager always keep high stock- level to make best use of profit of the inventory administration and depleting the order a new order begins. It might be profitable to raise the order level in each cycle and request a fresh order previous two stock runs out. Baker and Urban [2] designed an EOQ model with a store- linked, where the aim was profit maximization. Tripathi [3] analyzed an EOQ structure of items whose demand is a decreasing association of trade cost. Alfares [4] published the inventory model containing stock- echelon sensitive demand and a storage time- connected, carrying cost taking two times - linked holding cost functions. Tripathi and Mishra [5] described a production inventory model for time - associated demand. Time induced demand EOQ systems are established by Dave [6] and Maiti et al. [7]. Yang [8] described a structure under store- linked demand rate and stock- connected carrying cost under shortages. Several research papers published in this direction by Chang et al. [9], Soni and Shah [10], Tripathi [11],Teng et al. [12], Hsieh and Dye [13], Wee and Wang [14], Zhou et al. [15], Shah et al. [16] etc.

Deterioration of items is a major problem in the universe. Generally almost all items deteriorate with time. Deterioration means freshness decay of commodities. A normal common man declines his activity after passing the time and at last comes to the end. Tripathi and Pandey [17] studied an EOQ model to find the optimal total cost for price sensitive demand with Weibull distribution. Geetha and Kumar [18] addressed a model in which inventory cost will be lowered, if the seller can effectively reduce deterioration by improving the storage facility. Jaggi et al. [19] offered a model, including selling price dependent demand under deterioration. Mishra and Talati [20] proposed a single set- up multiple deliveries for fading stuff with fixed life time.Jani et al. [21] have studied an inventory policy for the item which has expiry date with two levels of trade credit depending on the quantity of order. It is considered that a supplier is ready to give a mutually agreed credit period to retailer only if the order quantity purchased by retailer is more than the predetermined quantity of ordered. Some researches in this in this direction are Chaudhury et al. [22], Duan et al. [23], Pervin et al. [24], Teng and Chang [25], Dye [26], Ghiami and Williams [27], Shah et al. [28] and others.

In the world economics, inflation and time value of capital cannot be unnoticed because of uncertainty of demand, weather, climate change abruptly, storm lock down (for example COVID-19), labor strike, flood etc. Buzacott [29] presented first an EOQ system by assuming inflationary effect on costs. Yang et al.[30] designed a variety of EOQ models with time unstable demand pattern under inflation. Sarkar and Moon [31] explored a manufacture EOQ model for random demand through cause of inflation.Chaudhari et al. [32] designed a single retailer and single product which deteriorates continuously for time dependent deteriorating item with seasonal demand, quadratic demand is debated here which is suitable for the items whose demand with starting of the season increases initially and after end of the season, it starts decreases. Reduction of deterioration is reduced by preservation technology. Some researchers like Sarkar et al. [33], Yang [34], Dey et al. [35], Tripathi and Chaudhary [36] consequences special type of structures under inflation and time - reduction.

Trade credits and shortages both play an important role in any type of business. Tripathi [37] published at an EOQ system for spoilage products with curvilinear time - linked demand, shortages under traffic credits. Pal and Chandra [38] established a sporadic review EOQ model under traffic credits and price discounts. Chern et al. [39] extended an EOQ system to include particulars that (i) advertising cost is considerably superior to unit acquiring cost and (ii) interest rate charged by the trader is not essentially advanced than the seller's savings return rate under permitted delay. Jiangtao et al. [40] addressed a multi- commodity system for unpreserved substances where demand rates of goods are stored- linked two- echelon 
traffic credits. Chern and Teng [41] designed an EOQ system for trader for finding his/ her best possible replenishment cycle time, included the fact that (i) failing foodstuffs decline constantly and having utmost life time and (ii) a seller frequently proposes an allowed delay in payments to draw additional purchasers.

The remainder of the work designed as follows. In the subsequent Sect. 2, assumptions and notation are mentioned. Mathematical formulation is argued in Sect. 3. The most beneficial explanation is renowned in part 4. After that, numerical examples are offered of all four cases to display hypothetical fallouts. Sensitivity exploration by means of distinct parameters is conversed in segment 6 . We present conclusion and future research in the last.

\section{Notations and Assumptions}

\section{Notations}

$K$ cost of ordering (in $\$$ )

$c, p, h$ and $s$ unit purchasing, selling, carrying and shortage cost/item

$Q_{1}$ and $Q_{2}$ highest inventory level and maximum shortage quantity

$Q$ Lot - size

$D(t)$ demand rate

$I_{p}$ and $I_{e}$ unit interest paid and earned $/ \$$

$Q(t)$ level of inventory at moment ' $t$ '

$\phi$ deterioration rate.

$r$ cash reduction rate, ' $r$ ' lies between zero and one

$t_{l}$ time to end up inventory

$M_{1}$ and $M_{2}$ stage of cash reduction and allowable delay $\left(M_{2}>M_{1}\right)$

$T$ cycle time.

$C_{H}, C_{D}$ and $C_{S}$ carrying, deterioration and shortages cost

$S_{R}$ sales profits

$I P_{1}$ and $I P_{3}$ interest payable (in $\$$ ) (cases 1 and 3)

$I E_{i}$ interest earned (in $\$$ ), $i=1-4$

$T_{i}{ }^{*}$ optimal $T$

$P_{i}(T)$ total profit /yr (in $\$$ )

\section{Assumptions}

1. Demand rate is time- sensitive, i.e. $D(t)=\alpha+\beta . t, \alpha$ is positive, $0 \leq \beta \leq 1, \beta$ is not zero.

2. Shortages are permitted.

3. Vendor suggests cash discount, if payment is ready in $t_{1}$, or as a well full expense is charged. Inside credit period $M_{2}$.

4. Replenishment arises immediately at endless pace.

5. Deterioration rate is steady, $0 \leq \phi<1$.

\section{Mathematical Models}

Reduction of originality is a shapeless and natural phenomenon for items with passing time. Some preservation technologies can maintain freshness for some time, but they cannot con- 
tinue for a long time. The level of inventory $Q(t)$ for some moment ' $t$ ' is ruled by subsequent equations are:

$$
\frac{d Q(t)}{d t}=\left\{\begin{array}{c}
-\phi Q(t)-D(t), 0 \leq t \leq t_{1} \\
-D(t), t_{1} \leq t \leq T
\end{array}\right.
$$

Solution of (1) and (2) with $Q\left(t_{1}\right)=0$ is:

$$
Q(t)=\left\{\begin{array}{c}
\frac{1}{\phi}\left\{(\alpha \phi-\beta)\left(\frac{e^{\phi\left(t_{1}-t\right)}-1}{\phi}\right)+\beta\left(t_{1} e^{\iota\left(t_{1}-t\right)}-t\right)\right\} \\
-\left(t-t_{1}\right)\left\{\alpha+\frac{\beta\left(t_{1}+t\right)}{2}\right\} \\
Q=\mathrm{Q}_{1}+Q_{2}
\end{array}\right.
$$

where $Q_{1}$ and $Q_{2}$ is obtained by substituting $\mathrm{t}=0$ and $\mathrm{t}=T$ in (4) and (5) respectively?

$$
\begin{gathered}
C_{H}=\frac{h}{\phi}\left\{\left(\alpha-\frac{\beta}{\phi}\right)\left(\frac{e^{\phi t_{1}}-1-\phi t_{1}}{\phi}\right)+\beta t_{1}\left(\frac{e^{\phi t_{1}}-1}{\phi}-\frac{t_{1}}{2}\right)\right\} \\
C_{S}=s \int_{t_{1}}^{T}-Q(t) d t=\frac{s\left(T-t_{1}\right)^{2}}{6}\left\{3 \alpha+\beta\left(T+2 t_{1}\right)\right\}
\end{gathered}
$$

Since seller offers permitted delay of cash reduction and payment. As a result, two situations may occur (i) payment is pleased at $M_{1}$ with reduction and (ii) payment is paid at $M_{l}$, lacking the cash diminish.

Case 1: $\mathrm{M}_{1} \leq \mathrm{t}_{1} \leq \mathrm{T}$

In nearby learning, $\mathrm{T} \geq M_{1}$. Since supplier presents allowed delay of cash concession, interest paid is nil. Figures of all mentions cases are as follows:

Therefore,

$$
\begin{gathered}
I P_{1}=\frac{c I_{p}}{\phi}\left[\left(\alpha-\frac{\beta}{\phi}\right)\left\{\frac{e^{\phi\left(t_{1}-M_{1}\right)}-1-\phi\left(t_{1}-M_{1}\right)}{\phi}\right\}+\beta\left\{\frac{t_{1}\left(e^{\phi\left(t_{1}-M_{1}\right)}-1\right)}{\phi}-\frac{\left(t_{1}^{2}-M_{1}^{2}\right)}{2}\right\}\right] \\
I E_{1}=p I_{c} \int_{0}^{M_{1}}(\alpha+\beta t) . t \cdot d t=p I_{e} M_{1}^{2}\left(\frac{\alpha}{2}+\frac{\beta M_{1}}{3}\right)
\end{gathered}
$$

And,

$$
S_{R}=p \int_{0}^{T}(\alpha+\beta t) d t=p T\left(\alpha+\frac{\beta T}{2}\right)
$$
Thus

The trader gets a cash allowance from supplier, due to payment is remunerated at $M_{l}$.

$$
C_{D}=\operatorname{rp}\left[\frac{1}{\phi}\left\{\left(\alpha-\frac{\beta}{\phi}\right)\left(e^{\phi t_{1}}-1\right)+\beta t_{1} e^{\phi t}\right\}+\frac{\left(T-t_{1}\right)\left\{2 \alpha+\beta\left(t_{1}+T\right)\right\}}{2}\right]
$$

Thus,

$$
P_{1}(T)=\left\{S_{R}-\left(A+C_{H}+C_{S}+I P_{1}-I E_{1}-C_{D}\right)\right\} / T
$$

Case 2: $\mathrm{M}_{1}>\mathrm{T}$

In this situation, $M_{1}>T$, therefore $I C_{2}=0$ and

$$
I E_{2}=p I_{e} t_{1}\left\{\alpha\left(M_{1}-\frac{t_{1}}{2}\right)+\frac{\beta t_{1}}{2}\left(M_{1}-\frac{t_{1}}{3}\right)\right\}
$$


Thus,

$$
P_{2}(T)=\left\{S_{R}-\left(A+C_{H}+C_{S}-I E_{2}-C_{D}\right)\right\} / T
$$

\section{Payment is Compensated at Credit Phase $M_{2}$}

Case 3: $\mathrm{M} 2<\mathrm{T}$

In such case, $T>M_{2}$, the seller has no cash price cut, thus

$$
\begin{gathered}
I P_{3}=c I_{p} \int_{M_{2}}^{t_{1}} Q(t) d t \\
I E_{3}=p I_{e} \int_{0}^{M_{2}}(\alpha+\beta t) t d t=p I_{e} M_{2}^{2}\left(\frac{\alpha}{2}+\frac{\beta M_{2}}{3}\right)
\end{gathered}
$$

As a result

$$
P_{3}(T)=\left\{S_{R}-\left(A+C_{H}+C_{S}+I P_{3}-I E_{3}\right)\right\} / T
$$

Case 4: $\mathrm{M} 2 \geq \mathrm{T}$

In this situation, $\mathrm{T} \leq M_{2}$, the vendor has no cash price cut $\& I P_{4}=0$, thus

$$
\begin{gathered}
I E_{4}=p I_{e} t_{1}\left\{\alpha\left(M_{2}-\frac{t_{1}}{2}\right)+\frac{\beta t_{1}}{2}\left(M_{2}-\frac{t_{1}}{3}\right)\right\} \\
P_{4}(T)=\left\{S_{R}-\left(A+C_{H}+C_{S}-I E_{4}\right)\right\} / T
\end{gathered}
$$

For small decline rate, we can presume (Figs. 1, 2, 3 and 4).

$$
e^{\phi T} \approx 1+\phi T+\frac{\phi^{2} T^{2}}{2} \text { etc, } \phi T<1
$$

Hence, the entirety income of case $1-4$ is falling too:

$$
\begin{gathered}
P_{1}=\frac{(1+r) p(2 \alpha+\beta T)}{2}-\frac{K}{T}-\frac{h t_{1}^{2}\left\{\alpha\left(3+\phi t_{1}\right)+t_{1}\left(2+\theta T_{1}\right) \beta\right\}}{6 T}- \\
\frac{s\left(T-t_{1}\right)^{2}\left\{3 \alpha+\beta\left(2 t_{1}+T\right)\right\}}{6 T}-\frac{c I_{p}\left(t_{1}-M_{1}\right)^{2}}{6 T} \\
{\left[\alpha\left\{3+\left(t_{1}-M_{1}\right) \phi\right\}+\beta\left\{M_{1}+2 t_{1}+\phi\left(t_{1}-M_{1}\right) t_{1}\right\}\right]} \\
+\frac{p I_{e} M_{1}^{2}}{6 T}\left(3 \alpha+2 \beta M_{1}\right)+\frac{r p \phi t_{1}^{2}\left(\alpha+\beta t_{1}\right)}{2 T} \\
P_{2}=p(1+r)\left(\alpha+\frac{\beta T}{2}\right)-\frac{K}{T}-\frac{h t_{1}^{2}\left\{a\left(3+\phi t_{1}\right)+t_{1}\left(2+\phi t_{1}\right) \beta\right\}}{6 T}-\frac{s\left(T-t_{1}\right)^{2}\left\{3 \alpha+\left(T+2 t_{1}\right) \beta\right\}}{6 T} \\
+\frac{p I_{e} t_{1}}{6 T}\left\{3 \alpha\left(2 M_{1}-t_{1}\right)+\beta t_{1}\left(3 M_{1}-t_{1}\right)\right\}+\frac{r p(2 \alpha+\beta T)}{2}+\frac{r p t_{1}^{2}\left(\alpha+\beta t_{1}\right) \phi}{2 T} \\
P_{3}=p\left(\alpha+\frac{\beta T}{2}\right)-\frac{K}{T}-\frac{h t_{1}^{2}\left\{\alpha\left(3+\phi t_{1}\right)+\beta\left(2+\phi t_{1}\right) t_{1}\right\}}{6 T}-\frac{s\left(T-t_{1}\right)^{2}\left\{3 \alpha+\beta\left(2 t_{1}+T\right)\right\}}{6 T} \\
-\frac{c I_{p}\left(t_{1}-M_{2}\right)^{2}\left[\alpha\left\{3+\phi\left(t_{1}-M_{2}\right)\right\}+\beta\left\{M_{2}+2 t_{1}+\phi\left(t_{1}-M_{2}\right) t_{1}\right\}\right]}{6 T}+\frac{p I_{e} M_{2}^{2}}{T}\left(\frac{\alpha}{2}+\frac{\beta M_{2}}{3}\right) \\
P_{4}=p\left(\alpha+\frac{\beta T}{2}\right)-\frac{K}{T}-\frac{h t_{1}^{2}}{6 T}\left\{\alpha\left(3+\phi t_{1}\right)+\left(2+\phi t_{1}\right) \beta t_{1}\right\}-\frac{s\left(T-t_{1}\right)^{2}\left\{3 \alpha+\beta\left(T+2 t_{1}\right)\right\}}{6 T}
\end{gathered}
$$




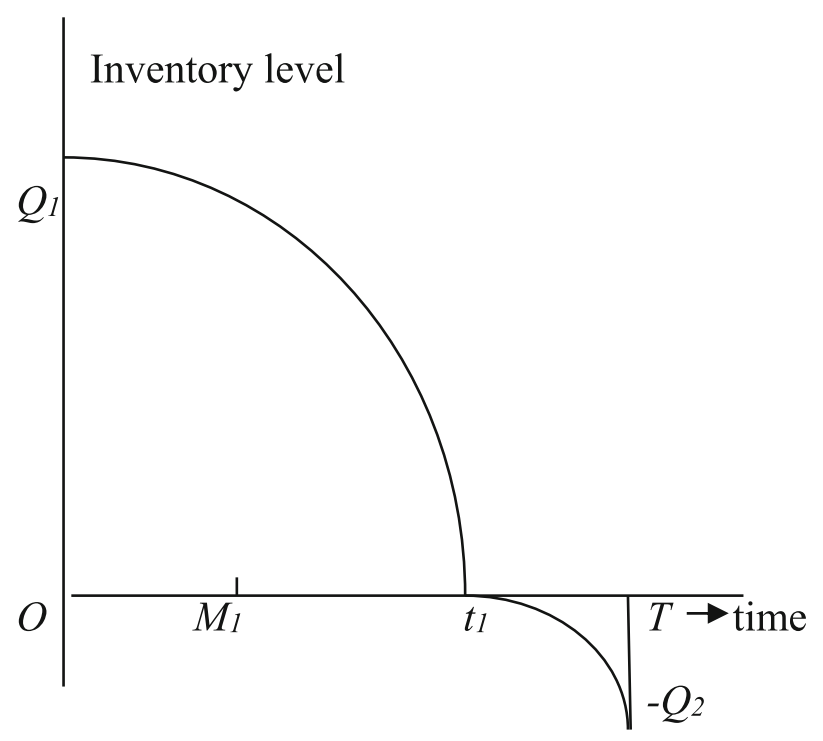

Fig. $1 \mathrm{M}_{1}<\mathrm{T}$

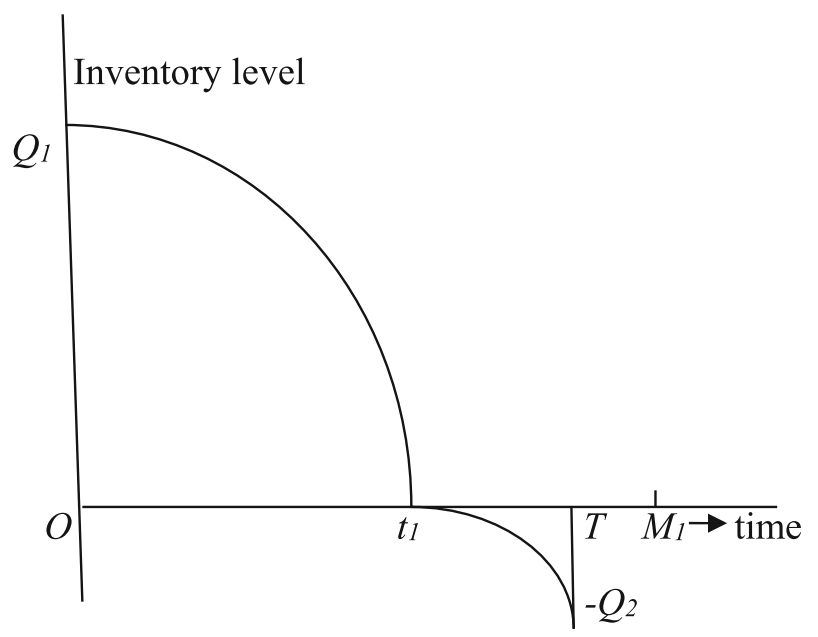

Fig. $2 \mathrm{M}_{1} \geq \mathrm{T}$

$$
+\frac{p I_{e} t_{1}}{6 T}\left\{3 \alpha\left(2 M_{2}-t_{1}\right)+\beta\left(3 M_{2}-t_{1}\right) t_{1}\right\}
$$

Since $t_{1}<T$, taking, $t_{1}=\gamma T, \gamma$ is stable $(0<\gamma<1)$. Equations (19) - (22) become:

$$
\begin{aligned}
P_{1}(T)= & \frac{p(1+r)(2 \alpha+\beta T)}{2}-\frac{K}{T}-\frac{h \gamma^{2}\{\alpha(3+\gamma \phi T)+\beta(2+\phi \gamma T) \gamma T\} T}{6} \\
& -\frac{s(1-\gamma)^{2}\{3 \alpha+(1+2 \gamma) \beta T\} T}{6} \\
& -\frac{c I_{p}\left(T \gamma-M_{1}\right)^{2}\left[\alpha\left\{3+\phi\left(\gamma T-M_{1}\right)\right\}+\beta\left\{M_{1}+2 T \gamma+\gamma \phi\left(T \gamma-M_{1}\right)\right\} T\right]}{3 T}
\end{aligned}
$$




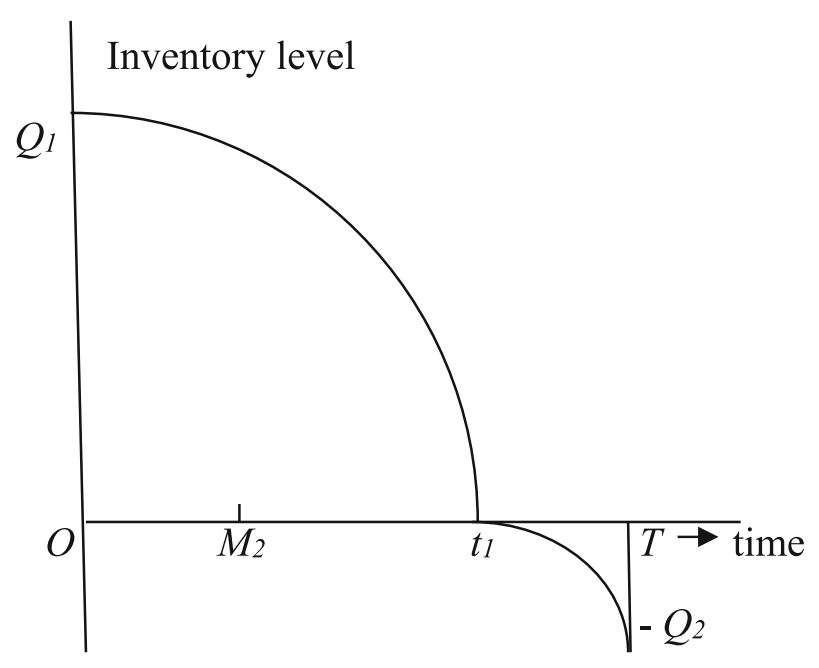

Fig.3 $M_{2}<T$

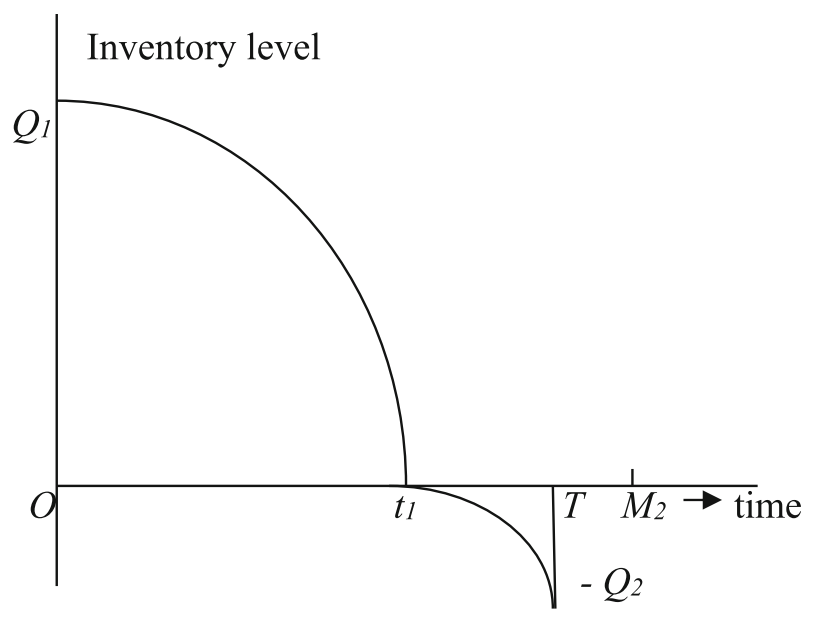

Fig. $4 M_{2} \geq T$

$$
\begin{aligned}
+ & \frac{p I_{e}\left(3 \alpha+2 \beta M_{1}\right) M_{1}^{2}}{6 T}+\frac{r p \gamma^{2}(\alpha+\beta \gamma T) T}{2} \\
P_{2}(T)= & \frac{(1+r) p(2 \alpha+\beta T)}{2}-\frac{K}{T}-\frac{h \gamma^{2}\{\alpha(3+\phi \gamma T)+\beta \gamma(2+\gamma \phi T) T\} T}{6} \\
& -\frac{s(1-\gamma)^{2}\{3 \alpha+\beta T(1+2 \gamma)\} T}{6} \\
& +\frac{p I_{e} \gamma\left\{3 \alpha\left(2 M_{1}-\gamma T\right)+\beta \gamma T\left(3 M_{1}-\gamma T\right)\right\}}{6}+\frac{r \phi \gamma^{2}(a+\beta \gamma T) p T}{2} \\
P_{3}(T)=p & \left(\alpha+\frac{\beta T}{2}\right)-\frac{K}{T}-\frac{h \gamma^{2}\{a(3+\phi \gamma T)+\beta(2+\gamma \phi T) \gamma T\} T}{6} \\
& -\frac{s(1-\gamma)^{2}\{3 \alpha+\beta(1+2 \gamma) T\} T}{6}
\end{aligned}
$$




$$
\begin{gathered}
-\frac{c I_{p}\left(\gamma T-M_{2}\right)^{2}}{6 T}\left[\alpha\left\{3+\left(\gamma T-M_{2}\right) \phi\right\}+\beta\left\{M_{2}+2 \gamma T+\gamma \phi\left(\gamma T-M_{2}\right) T\right\}\right] \\
+\frac{p I_{e}\left(3 \alpha+2 \beta M_{2}\right) M_{2}^{2}}{6 T} \\
P_{4}(T)=\frac{p(2 \alpha+\beta T)}{2}-\frac{K}{T}-\frac{h \gamma^{2} T\{\alpha(3+\phi \gamma T)+\beta \gamma(2+\phi \gamma T) T\}}{6} \\
\quad-\frac{s(1-\gamma)^{2}\{3 \alpha+\beta(2 \gamma+1) T\} T}{6} \\
\quad+\frac{p I_{e} \gamma\left\{3 \alpha\left(2 M_{2}-\gamma T\right)+\beta \gamma T\left(3 M_{2}-\gamma T\right)\right\}}{6}
\end{gathered}
$$

\section{Optimal Solution}

Necessary and sufficient circumstances for maximization are: $\frac{d P_{i}}{d T}=0$, and $\frac{d^{2} P_{i}}{d T^{2}}<0 . P_{i}$, for $i=1-4$.

Putting first derivative of (23) - (26) w.r.t. $T$, to zero, we find

$$
\begin{aligned}
& 3 \beta(1+r) p T^{2}+6 K-h \gamma^{2} T^{2}\{\alpha(3+2 \gamma \phi T)+\beta(4+3 \gamma \phi T) \gamma T\}-s(1-\gamma)^{2}\{3 \alpha+2(1+2 \gamma) \beta T\} T^{2} \\
& -c I_{p}\left\{(\gamma T)^{2}-M_{1}^{2}\right\}\left[\alpha\left\{3+\phi\left(T \gamma-M_{1}\right)\right\}+\left\{M_{1}+2 T \gamma+\phi \gamma\left(\gamma T-M_{1}\right) T\right\} \beta\right] \\
& -c I_{p} \gamma\left(\gamma T-M_{1}\right)^{2} T\left[\alpha \phi+\beta\left\{2+\left(2 T \gamma-M_{1}\right) \phi\right\}\right]-p I_{e}\left(3 \alpha+2 \beta M_{1}\right) M_{1}^{2}+r p \gamma^{2} \phi(\alpha+2 \beta \gamma T) T^{2}=0
\end{aligned}
$$$$
6 \beta(1+r) p T^{2}+6 K-h \gamma^{2} T^{2}\{\alpha(3+2 \gamma \phi T)+\gamma \beta(4+3 \phi \gamma T) T\}-s(1-\gamma)^{2}\{3 \alpha+2 \beta(1+2 \gamma) T\} T^{2}
$$$$
{ }_{-} p I_{e} \gamma^{2}\left\{3 \alpha-\beta\left(3 M_{1}-2 \gamma T\right)\right\} T^{2}+r p \phi(\gamma T)^{2}(\alpha+2 \gamma \beta T)=0
$$

$3 \beta p T^{2}-h \gamma^{2} T^{2}\{\alpha(3+2 \gamma \phi T)+\beta \gamma T(4+3 \gamma \phi T)\}-s(1-\gamma)^{2}\{3 \alpha+2 \beta(1+2 \gamma) T\} T^{2}$

$-c I_{p}\left(T^{2} \gamma^{2}-M_{2}^{2}\right)\left[\alpha\left\{3+\phi\left(\gamma T-M_{2}\right)\right\}+\beta\left\{M_{2}+2 \gamma T+\phi \gamma\left(\gamma T-M_{2}\right) T\right\}\right]$

$-c I_{p} \gamma T\left(\gamma T-M_{2}\right)^{2}\left[\alpha \phi+\beta\left\{2+\phi\left(2 \gamma T-M_{2}\right)\right\}\right]-p I_{e} M_{2}^{2}\left(3 \alpha+2 \beta M_{2}\right)$

$-3 c I_{p}\left(t_{1}-M_{2}\right)^{2}\left(\alpha+\beta t_{1}\right)-p I_{e} M_{2}^{2}\left(3 \alpha+2 \beta M_{2}\right)+6 K=0$

and

$$
\begin{aligned}
& 3 \beta p T^{2}+6 K-h \gamma^{2}\{\alpha(3+2 \gamma \phi T)+\beta \gamma(4+3 \gamma \phi T) T\} T^{2}-s(1-\gamma)^{2}\{3 \alpha+2(1+2 \gamma) \beta T\} T^{2} \\
& +p I_{e} \gamma^{2} T^{2}\left\{3 \alpha-\left(3 M_{2}-2 \gamma T\right) \beta\right\}=0
\end{aligned}
$$

Also

$$
\begin{gathered}
\frac{d^{2} P_{1}}{d T^{2}}=-\frac{2 K}{T^{3}}-\frac{h \gamma^{3}\{\alpha \phi+\beta(2+3 \phi \gamma T\}}{3}-\frac{s \beta(1-\gamma)^{2}(2 \gamma+1)}{3} \\
-\frac{c I_{p}\left(\gamma^{2} T^{2}-M_{1}^{2}\right)\left[\alpha \phi+\beta\left\{2+\phi\left(2 \gamma T-M_{1}\right)\right\}\right]}{3} \\
\frac{c I_{p} M_{1}^{2}}{T^{3}}\left[\alpha\left\{1+\frac{\phi\left(\gamma T-M_{1}\right)}{3}\right\}+\left\{M_{1}+2 \gamma T+\phi \gamma\left(\gamma T-M_{1}\right) T\right\}\right]+\frac{c I_{p} \lambda^{2} \beta \phi}{T^{3}}+r \beta p \phi \gamma^{3} \\
\frac{d^{2} P_{2}}{d T^{2}}=-\left[\frac{2 K}{T^{3}}+\frac{h \gamma^{3}\{\alpha \phi+\beta(2+3 \phi \gamma T\}}{3}+\frac{s \beta(1-\gamma)^{2}(1+2 \gamma)}{3}+\frac{\beta p I_{e} \gamma^{3}}{3}-r \beta p \phi \gamma^{3}\right]<0
\end{gathered}
$$


Fig. 5 Figure between $T \& P_{1}$

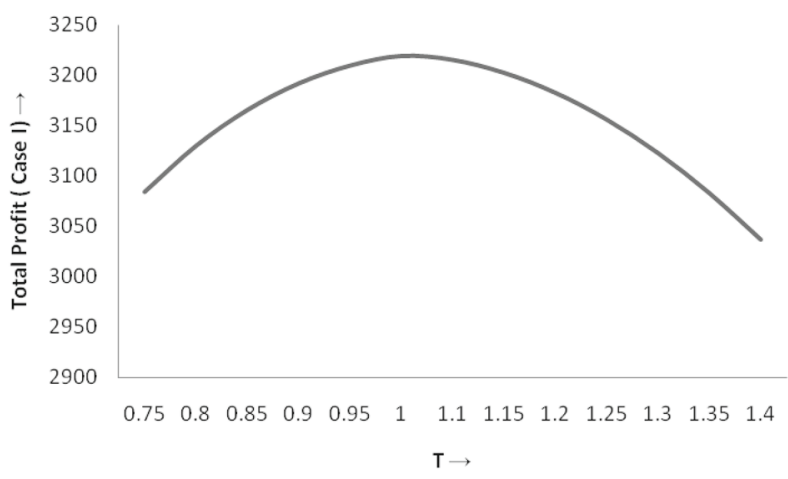

Fig. 6 Illustrative depiction

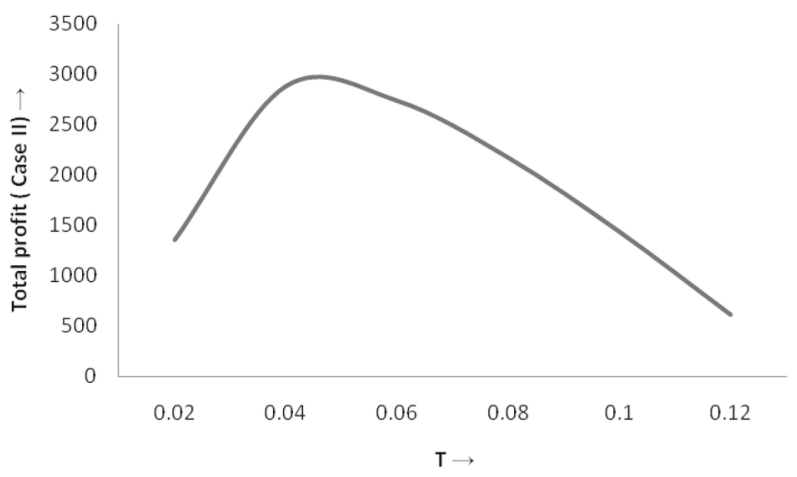

connecting $T \& P_{2}$

$$
\begin{aligned}
& \frac{d^{2} P_{3}}{d T^{2}}=-\frac{2 K}{T^{3}}-\frac{h \gamma^{3}\{\alpha \phi+\beta(2+3 \phi \gamma T\}}{3}-\frac{s \beta(1-\gamma)^{2}(1+2 \gamma)}{3} \\
& -\frac{c I_{p}\left(\gamma^{2} T^{2}-M_{2}^{2}\right)\left[\alpha \phi+\beta\left\{2+\phi\left(2 \gamma T-M_{2}\right)\right\}\right]}{3} \\
& -\frac{c I_{p} M_{2}^{2}}{T^{3}}\left[\alpha\left\{1+\frac{\left(\gamma T-M_{2}\right) \phi}{3}\right\}+\left\{M_{2}+2 \gamma T+\gamma \phi T\left(\gamma T-M_{2}\right)\right\}\right] \\
& -\frac{c I_{p} \gamma^{2} \beta \phi}{3 T}+\frac{2 p I_{e} M_{2}^{2}}{T^{3}}\left(\frac{\alpha}{2}+\frac{\beta M_{2}}{3}\right)<0 \\
\frac{d^{2} P_{4}}{d T^{2}}=- & -\left[\frac{2 K}{T^{2}}+\frac{h \gamma^{3}\{\alpha \phi+\beta(2+3 \gamma \phi T)\}}{3}-\frac{s(1-\gamma)^{2}(1+2 \gamma)}{3}+\frac{\beta p I_{e} \gamma^{3}}{3}\right]<0
\end{aligned}
$$

Since $\frac{d^{2} P_{i}}{d T^{2}}<0, \mathrm{i}=1-4 . P_{i}$ is maximum at $T_{i}{ }^{*}$. We have also shown by graphs in numerical examples.

\section{Algorithm}

In this section, we provide a solution procedure and flow diagram for finding an optimal resolution.

Step 1 locate $T_{i} *$ by resolve (27)-(30), $i=1-4$.

Step 2 if $T_{1} * \geq M_{1}$, come across $P_{1} *$ by (23).

Step 3 if $T_{2} *<M_{1}$, discover $P_{2} *$ by (24). 
Fig. 7 Pictographic demonstration connecting $T$ and $P_{3}$

Fig. 8 Graphical depiction $T$ vs. $\mathrm{P}_{4}$
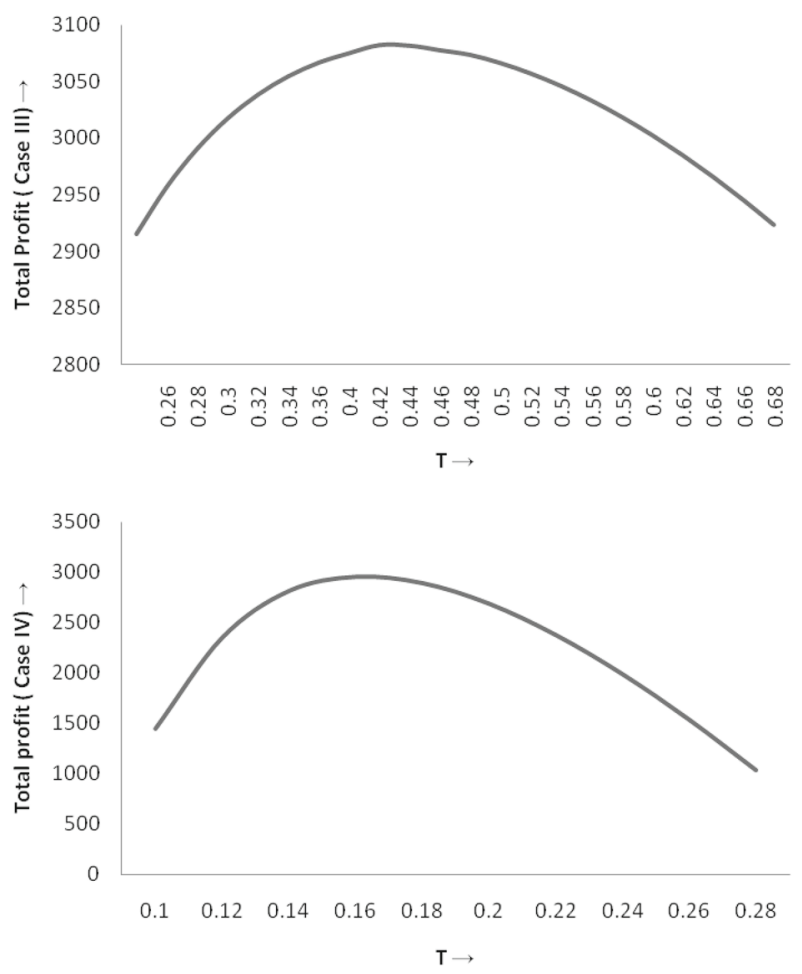

Step 4 if $T_{3} * \geq M_{2}$, find $P_{3} *$ by (25).

Step 5 if $T_{4} *<M_{2}$, locate $P_{4} *$ by (26).

Step 6 Obtain most favorable income $P_{i}=\max P_{i}$.

Step 7 end.

\section{Numerical Examples}

Examples are supplied to make obvious conclusions of structure discussed in each case:

Example $1\left(M_{1} \leq \mathrm{T}\right)$.

Bearing in mind subsequent constrains in proper component:

$\alpha=1.5 \times 10^{3}, \beta=150, \phi=1 / 100, s=100, K=1.0 \times 10^{3}, p=250, h=10, I_{e}=$ $13 / 100, I_{p}=3 / 20, M_{1}=1 / 5 \mathrm{yr}, c=100, r=1 / 50 \& \gamma=3 / 5$. Putting these in (27), and resolving for $T$, we find, $T_{1} *=0.65989 \mathrm{yr}$, that validate case 1 , cprresponding $Q^{*}=1023.7$ and $P_{1} *=\$ 2973.7$.

Example $2\left(M_{1}>\mathbf{T}\right)$.

Considering following strictures in their suitable units:

$\alpha=1.5 \times 10^{3}, \beta=150, \phi=1 / 100, \mathrm{~s}=500, h=10, K=100, p=300, I_{e}=13 / 100, I_{p}$ $=3 / 20, M_{1}=1 / 5 \mathrm{yr}, c=50, r=1 / 50 \& \gamma=3 . / 5$. Replacement of those in (28) and solving for $T$, we gain, $T_{2} *=0.1157 \mathrm{yr}$, which proves case 2 , accordingly $Q^{*}=174.57 \& P_{2} *=\$$ 794.37 . 
Table 1 Deviation of $T, Q$ and $P_{i}$ by $s, K, c, p, h, \phi, \alpha$ and $\beta$

\begin{tabular}{llllllll}
\hline$s$ & $T^{*}$ & $Q^{*}$ & $P_{1}^{*}$ & $K$ & $T^{*}$ & $Q^{*}$ & $P_{1}^{*}$ \\
\hline 105 & 0.55973 & 863.97 & 2448.7 & 1100 & 0.69855 & 1085.8 & 2882.8 \\
110 & 0.48786 & 750.29 & 2020.7 & 1200 & 0.73295 & 1141.2 & 2793.4 \\
115 & 0.43481 & 666.92 & 1717.6 & 1300 & 0.76408 & 1191.6 & 2705.4 \\
120 & 0.39437 & 603.65 & 1347.1 & 1400 & 0.79263 & 1237.8 & 2619.0 \\
125 & 0.36259 & 554.11 & 1069.4 & 1500 & 0.81908 & 1280.8 & 2533.9 \\
\hline$c$ & $T^{*}$ & $Q^{*}$ & $P_{1} *$ & $p$ & $T^{*}$ & $Q^{*}$ & $P_{1}^{*}$ \\
\hline 105 & 0.63346 & 981.41 & 2903.7 & 260 & 0.80909 & 1264.6 & 3860.7 \\
110 & 0.61039 & 944.57 & 2842.4 & 270 & 1.01837 & 1608.3 & 4993.1 \\
115 & 0.59018 & 912.36 & 2788.4 & 280 & 1.27802 & 2044.3 & 6417.3 \\
120 & 0.57241 & 884.10 & 2740.8 & 290 & 1.56867 & 2544.8 & 8148.3 \\
125 & 0.55671 & 859.18 & 2698.5 & 300 & 1.87570 & 3088.0 & 9976.4 \\
\hline$h$ & $T^{*}$ & $Q^{*}$ & $P_{1} *$ & $\phi$ & $T^{*}$ & $Q^{*}$ & $P_{1}^{*}$ \\
\hline 12 & 0.57088 & 881.67 & 2505.4 & 0.02 & 0.65874 & 1023.2 & 2969.2 \\
14 & 0.50394 & 775.66 & 2113.6 & 0.03 & 0.65771 & 1022.7 & 2964.8 \\
16 & 0.45278 & 695.11 & 1777.9 & 0.04 & 0.65664 & 1022.1 & 2960.4 \\
18 & 0.41281 & 632.46 & 1483.8 & 0.05 & 0.65559 & 1021.6 & 2956.0 \\
20 & 0.38084 & 582.53 & 1221.2 & 0.06 & 0.65454 & 1021.2 & 2951.7 \\
1250 & 1.8827 & 2533.8 & 8242.99 & 200 & 2.24652 & 3890.5 & $13,933.5$ \\
\hline$\alpha$ & $T^{*}$ & $Q^{*}$ & $P_{1}^{*}$ & $\beta$ & $T^{*}$ & $Q^{*}$ & $P_{1}^{*}$ \\
\hline 1000 & 2.9219 & 3581.6 & $14,854.0$ & 160 & 0.92394 & 1456.7 & 4323.9 \\
1050 & 2.6585 & 3337.9 & $12,968.5$ & 170 & 1.26014 & 2029.8 & 6151.1 \\
1100 & 2.3968 & 3080.9 & $11,238.0$ & 180 & 1.60881 & 2654.0 & 8405.0 \\
1200 & 2.1378 & 2812.2 & 9662.94 & 190 & 1.94021 & 3279.6 & $11,017.0$ \\
\hline & 1.8827 & 2533.8 & 8242.99 & 200 & 2.24652 & 3890.5 & $13,933.5$ \\
\hline
\end{tabular}

Example $3\left(M_{2}<\mathbf{T}\right)$.

Let us choose following constraints in proper entities:

$\alpha=1.1 \times 10^{3}, \beta=150, \phi=1 / 100, s=100, K=1.0 \times 10^{3}, p=250, h=10, I_{e}=$ $13 / 100, I_{p}=3 / 20, M_{1}=1 / 4 \mathrm{yr}, c=100, r=1.50$, and $M_{2}=140$ days. On putting these (29) and solving for $T$, we find, $T_{3} *=0.42588 \mathrm{yr}$, which confirms case 3 , related $Q^{*}=652.92$ $\& P_{3} *=\$ 3082.5$.

\section{Example $4\left(M_{2} \geq \mathbf{T}\right)$.}

Following constraints are taken in suitable units:

$\alpha=1.5 \times 10^{3}, \beta=150, \phi=1 / 100, s=100, K=1.0 \times 10^{3}, p=500, h=10, I_{e}=$ $13 / 100, I_{p}=3 / 20, c=50, r=1 / 100$, and $M_{2}=1 / 4 \mathrm{yr}$. On substituting those in (30), and solving for $T$, we obtain, $T_{4} *=0.19933$ years, that verifies case 4 , resultant $Q^{*}=338.0 \&$ $P_{4} *=\$ 2328.2$.

Using above algorithm Case 3 gives the optimal (maximum) solution (Figs. 5, 6, 7 and 8). 
Table 2 Disparity of $T, Q$ and $P_{i}$ with $s, K, p, h, \alpha$, and $\beta$

\begin{tabular}{llllllll}
\hline$s$ & $T^{*}$ & $Q^{*}$ & $P_{2} *$ & $K$ & $T^{*}$ & $Q^{*}$ & $P_{2} *$ \\
\hline 510 & 0.10770 & 162.46 & 995.48 & 105 & 0.11837 & 178.65 & 639.13 \\
520 & 0.10111 & 152.47 & 1146.3 & 110 & 0.12098 & 182.60 & 467.84 \\
530 & 0.09557 & 144.07 & 1260.5 & 115 & 0.12352 & 186.46 & 340.12 \\
540 & 0.09083 & 136.89 & 1347.3 & 120 & 0.12599 & 190.22 & 195.83 \\
\hline$s$ & $T^{*}$ & $Q^{*}$ & $P_{2} *$ & $K$ & $T^{*}$ & $Q^{*}$ & $P_{2}^{*}$ \\
\hline$p$ & $T^{*}$ & $Q^{*}$ & $P_{2} *$ & $h$ & $T^{*}$ & $Q^{*}$ & $P_{2} *$ \\
280 & 0.09515 & 143.43 & 1044.4 & 12 & 0.11192 & 168.85 & 891.37 \\
285 & 0.09929 & 149.71 & 1025.6 & 14 & 0.10848 & 163.62 & 976.45 \\
290 & 0.10440 & 156.84 & 982.16 & 16 & 0.10532 & 158.84 & 1032.2 \\
295 & 0.10941 & 165.04 & 907.87 & 18 & 0.10241 & 154.43 & 1117.2 \\
310 & 0.15204 & 199.42 & 397.41 & 20 & 0.09972 & 150.36 & 1175.4 \\
\hline$\alpha$ & $T^{*}$ & $Q^{*}$ & $P_{2} *$ & $\beta$ & $T^{*}$ & $Q^{*}$ & $P_{2} *$ \\
\hline 1600 & 0.20466 & 330.71 & 2508.0 & 120 & 0.07868 & 118.40 & 1933.9 \\
1700 & 0.19034 & 326.41 & 2584.1 & 125 & 0.08253 & 124.24 & 1874.5 \\
1800 & 0.17865 & 324.06 & 2865.0 & 130 & 0.08699 & 130.99 & 1771.0 \\
1900 & 0.16886 & 323.07 & 3171.3 & 135 & 0.09222 & 138.93 & 1663.0 \\
2000 & 0.16051 & 323.05 & 3501.8 & 140 & 0.09848 & 148.42 & 1524.6 \\
\hline & & & & & & & \\
\hline
\end{tabular}

\section{Sensitivity Analysis}

\section{Case 1}

Considering identical data as in Ex. 1, sensitivity study is conversed. Fallouts are reviewed in Table 1.

\section{Case 2}

Using the same figures as in Ex. 2, sensitivity scrutiny is conversed in Table 2.

\section{Case 3}

With parallel information as in Ex 3, sensitivity inquiry id discussed below:

\section{Case 4}

By means of alike data as design in Ex. 4, sensitivity inspection is as follows:

Following judgment can be finished from Table 1:

1. Enlarge of $s, K, c$ and it will cause a drop of $P_{l}$.

2. Elevate of ' $p$ ' and $\phi$ will lead augment in $P_{1}$.

Following submission can be equipped from Table 2.

1. Lift of $s, K$ and $h$ will cause weakening in $P_{2}$. 
Table 3 Dissimilarity of $T, Q$ and $P_{i}$ by $s, K, c, p, h, \alpha . \beta$ and $\phi$

\begin{tabular}{llllllll}
\hline$s$ & $T^{*}$ & $Q^{*}$ & $P_{3}^{*}$ & $K$ & $T^{*}$ & $Q^{*}$ & $P_{3}^{*}$ \\
\hline 75 & 1.67627 & 2733.5 & 6987.5 & 1100 & 0.49783 & 766.03 & 2866.4 \\
80 & 1.31673 & 2110.2 & 5093.2 & 1200 & 0.55300 & 853.29 & 2676.3 \\
85 & 1.00272 & 1582.4 & 4340.7 & 1300 & 0.59869 & 925.92 & 2502.7 \\
90 & 0.74353 & 1158.3 & 3786.5 & 1400 & 0.63815 & 988.91 & 2341.1 \\
95 & 0.55171 & 857.21 & 3383.2 & 1500 & 0.67315 & 1045.0 & 2186.6 \\
\hline$c$ & $T^{*}$ & $Q^{*}$ & $P_{3}^{*}$ & $p$ & $T^{*}$ & $Q^{*}$ & $P_{3}^{*}$ \\
\hline 105 & 0.42468 & 651.04 & 3082.9 & 260 & 0.52326 & 806.18 & 3576.3 \\
110 & 0.42375 & 649.59 & 3082.4 & 270 & 0.60849 & 941.53 & 3847.7 \\
115 & 0.42302 & 648.50 & 3082.4 & 280 & 0.72163 & 1123.0 & 4148.2 \\
120 & 0.42342 & 647.51 & 3082.4 & 290 & 0.85594 & 1340.9 & 4487.9 \\
125 & 0.42193 & 646.74 & 3082.4 & 300 & 1.00290 & 1582.7 & 4874.5 \\
\hline$h$ & $T^{*}$ & $Q^{*}$ & $P_{3}^{*}$ & $\alpha$ & $T^{*}$ & $Q^{*}$ & $P_{3}^{*}$ \\
\hline 4 & 0.88331 & 1385.7 & 4114.6 & 1050 & 2.4952 & 2838.8 & 9524.6 \\
5 & 0.77881 & 1215.4 & 3882.6 & 1100 & 2.2308 & 2563.1 & 8170.0 \\
6 & 0.68476 & 1063.6 & 3679.2 & 1150 & 1.9681 & 2407.9 & 6982.0 \\
7 & 0.60240 & 1024.3 & 3501.0 & 1200 & 1.7079 & 1978.6 & 5959.8 \\
8 & 0.53225 & 820.04 & 3344.5 & 1250 & 1.4518 & 1675.3 & 5101.8 \\
\hline$\beta$ & $T^{*}$ & $Q^{*}$ & $P_{3}^{*}$ & $\phi$ & $T^{*}$ & $Q^{*}$ & $P_{3}^{*}$ \\
\hline 160 & 0.68667 & 1019.1 & 3016.7 & 0.03 & 0.42441 & 651.61 & 3080.5 \\
170 & 1.05000 & 1671.9 & 4298.9 & 0.04 & 0.42368 & 650.97 & 3079.4 \\
160 & 1.41903 & 2315.8 & 5859.2 & 0.05 & 0.42296 & 650.33 & 3078.5 \\
190 & 1.76202 & 2947.4 & 7735.1 & 0.06 & 0.42239 & 649.69 & 3077.5 \\
200 & 2.07512 & 2556.9 & 9809.1 & 0.07 & 0.42153 & 649.06 & 3076.5 \\
\hline & & & & & & & \\
\hline
\end{tabular}

2. Raise of $p$ consequences enhances $P_{2}$.

Following proposition can be finished from Table 3 .

1. Boost of $s, K$, and $h$ will direct diminish $P_{3}$.

2. Augment of will direct decline in $P_{3}$.

3. Amplification of $p$ causes moves up in $P_{3}$.

Deductions made from Table 4 are as follows:

1. Boost of $s$ and $p$ will express make bigger in $P_{4}$.

2. Improve of $A$ and $h$ lead, turn down in $P_{4}$.

\section{Managerial Insights}

These above deviations have the following managerial implications.

- Higher values of $s, c, h, \phi$ and $\alpha$ implies lower values of cycle time, order quantity and total profit for case I and III. 
Table 4 Discrepancy of $T, Q \& P_{i}$ by $s, K, c, p$ and $h$

\begin{tabular}{llllllll}
\hline$s$ & $T$ & $Q^{*}$ & $P_{4}^{*}$ & $K$ & $T^{*}$ & $Q^{*}$ & $P_{4}^{*}$ \\
\hline 460 & 0.21621 & 327.94 & 2178.7 & 950 & 0.21711 & 329.32 & 2657.3 \\
470 & 0.21024 & 318.80 & 3026.5 & 960 & 0.21824 & 331.06 & 2590.9 \\
480 & 0.20475 & 310.39 & 1872.6 & 970 & 0.21837 & 332.80 & 2524.7 \\
490 & 0.19968 & 302.62 & 1717.4 & 980 & 0.22050 & 334.52 & 2454.9 \\
500 & 0.19498 & 285.42 & 1561.5 & 990 & 0.22162 & 336.24 & 2393.4 \\
\hline$p$ & $T^{*}$ & $Q^{*}$ & $P_{4}^{*}$ & $h$ & $T^{*}$ & $Q^{*}$ & $P_{4}^{*}$ \\
\hline 450 & 0.20427 & 306.45 & 700.10 & 12 & 0.21976 & 333.39 & 2261.4 \\
460 & 0.20758 & 314.72 & 1022.0 & 14 & 0.21691 & 329.02 & 2194.0 \\
470 & 0.21107 & 320.06 & 1361.2 & 16 & 0.21417 & 324.81 & 2126.1 \\
480 & 0.21474 & 325.70 & 1687.2 & 18 & 0.21153 & 320.80 & 2052.7 \\
490 & 0.21862 & 331.65 & 2009.7 & 20 & 0.20899 & 316.87 & 1989.0 \\
\hline$\alpha$ & $T^{*}$ & $Q^{*}$ & $P_{3}^{*}$ & $\beta$ & $T^{*}$ & $Q^{*}$ & $P_{3}^{*}$ \\
\hline 1600 & 0.20466 & 330.71 & 2584.1 & 100 & 0.17645 & 266.32 & 727.25 \\
1700 & 0.19034 & 326.41 & 2865.0 & 110 & 0.18345 & 277.12 & 1047.4 \\
1800 & 0.17865 & 324.06 & 3171.4 & 120 & 0.19134 & 289.31 & 1369.4 \\
1900 & 0.16886 & 323.07 & 3501.8 & 130 & 0.20032 & 303.20 & 1691.9 \\
2000 & 0.16051 & 232.05 & 3854.8 & 140 & 0.21065 & 319.21 & 2012.7 \\
\hline
\end{tabular}

- Higher values of $p$ and $\beta$ implies higher values of cycle time, order quantity and total profit for case I and III.

- Higher values of $\mathrm{K}$ implies higher values of cycle time and order quantity while lower values of total profit.

- Higher values of $s, h$, and $\alpha$ implies lower values of cycle time,order quantity and lower values total profit for case II and IV.

- Higher values of $K, p$, and $\beta$ implies higher values of cycle time,order quantity and lower values total profit for case II and IV.

\section{Conclusion}

In this study, we have deliberated EOQ models under trade credits permit for four unlike conditions. We have attempted to locate characteristic of cash decline into the conventional model with permitted delay. Numerical examples are completed on credible attempt. Optimal explanation is acquired for finding optimal variables. Solution process is communicated to find most advantageous solution. Sensitivity reading of the clarification for dissimilar constraints has been conferring. This research is obliging for returning products since demand for continuing foodstuffs is usually time allied. It is seen that disparity in shortage, ordering, procure, carrying and selling costs, lead to momentous possessions on finest $P_{i}, i=1-4$ ). Entire income is around stable with adjust in weakening rate. Outcomes came into view in sensitivity analysis is conflicting, like expand in cost fallouts reject of earnings whereas intensify in selling price argued lift in income.

A variety of likely extensions of the model that can be presented as like: (i) variable decay and Weibull deterioration (ii) to assume a variable carrying cost (iii) to comprise fall in the 
purchasing cost/ unit (iv) to study the case of inflation and shipment charges and (v) to study stock- sensitive demand.

\section{References}

1. Silver, E.S., Peterson, R.: Decision systems for inventory management and production planning, 2 nd edn. Wiley, New York (1995)

2. Baker, R.C., Urban, T.L.: A deterministic inventory system with an inventory level- dependent demand. Euro. J. Oper. Res. 39, 823-831 (1988)

3. Tripathi, R.P.: Economic order Quantity models for price dependent demand and different holding cost functions. Jor. J. Math. Stat. 12(1), 15-33 (2019)

4. Alfares, H.K.: Inventory model with stock- level dependent demand rate and variable holding cost. Int. J. Prod. Eco. 108, 259-265 (2007)

5. Tripathi, R.P., Mishra, S.: Innovative study of EPQ model with time - induced demand under decline release. Int. J. Sup. Chain. Oper. Manag. 6(1), 264-275 (2019)

6. Dave, U.: An order level inventory model for deteriorating items with variable instantaneous and discrete opportunities for replenishment. Opsearch 23, 244-254 (1986)

7. Maiti, A.K., Maiti, M.K., Maiti, M.: Inventory model with stochastic lead time and price dependent demand incorporating advance payment. App. Math. Mod. 33, 2433-2443 (2009)

8. Yang, C.T.: An inventory model with both stock- dependent demand rate and stock- dependent holding cost rate. Int. J. Pro. Eco. 155, 214-221 (2014)

9. Chang, C.T., Teng, J.T., Goyal, S.K.: Optimal replenishment policies for non-instantaneous deteriorating item with stock- dependent demand. Int. J. Pro. Eco. 123, 62-68 (2010)

10. Soni, H., Shah, N.H.: Optimal Ordering policy for stock- dependent demand under progressive payment scheme. Eur. J. Oper. Res. 184, 91-100 (2008)

11. Tripathi, R.P.: Inventory model with time - dependent demand rate under inflation when supplier credit linked to order quantity. Int. J. Bus. Inf. Tech. 1(3), 174-18 (2011)

12. Teng, J.T., Min, J., Pan, Q.: Economic order quantity model with trade credit financing for non- decreasing demand. Omega 40, 328-335 (2012)

13. Hsieh, T.P., Dye, C.Y.: A production - inventory model incorporating the effect of preservation technology investment when demand is fluctuating with time. J. App. Comp. Math. 239, 25-36 (2003)

14. Wee, H.M., Wang, W.T.: A variable production scheduling policy for deteriorating item with time- varying demand. Com. Oper. Res. 26(3), 237-254 (1999)

15. Zhau, Y.W., Lau, H.S., Yang, S.L.: A new variable production scheduling strategy for deteriorating item with time varying demand and partial lost sale. Com. Oper. Res 30(21), 1753-1776 (2003)

16. Shah, N.H., Chaudhari, U., Jani, M.Y.: An inventory model with an expiration date of items and deterioration under two-level trade credit and preservation technology investment for time and price-sensitive demand: DCF approach. Int. J. Log. Sys. Manag. 27(4), 420-437 (2017)

17. Tripathi, R.P., Pandey, H.S.: Optimal ordering policies for non- instantaneous Weibull deteriorating items with price linked demand under trade credits. Int. J. Sup. Cha. Inv. Mana. 3(2), 77-92 (2020)

18. Geetha, K.V., Uttayakumar, R.: Economic design for an inventory policy for non- instantaneous deteriorating item under permissible delay in payments. J. App. Comp. Math. 233(10), 2492-2505 (2020)

19. Jaggi, C.K., Tiwari, S., Goel, S.K.: Credit financing in economic ordering policies for non- instantaneous deteriorating item with price dependent demand and two storage facilities. Ann. Oper. Res. 248(1-2), 253-280 (2017)

20. Mishra, P., Talati, I.: Quantity discount for integrated supply chain model with back order and controllable deterioration rate. Yugo. J. Oper. Res. 28(5), 355-369 (2018)

21. Jani, MY., Shah, NH., and Chaudhari, UB.,: Inventory Control Policies for Time-Dependent Deteriorating Item with Variable Demand and Two Level Order Linked Trade Credit. In: Shah, NH., and Mittal, M., (eds.) Optimization and inventory management, (pp. 55-67). Springer, Singapore (2020)

22. Chaudhury, K.D., Karmakar, B., Das, M., Datta, T.K.: An inventory model for deteriorating items with stock- dependent demand, time varying holding cost and shortages. Opsearch 52(1), 55-74 (2015)

23. Duan, Y., Li, G., Tien, J.M., Huo, J.: Inventory models for perishable items with inventory level dependent demand rate. Appl. Math. Model. 36, 5015-5028 (2017)

24. Pervin, M., Roy, S.K., Weber, G.W.: A two - echelon inventory model with stock- dependent demand and variable holding cost for deteriorating item. Numer. Alg. Con. Opt. 7(1), 21-50 (2017)

25. Teng, J.T., Chang, C.T.: Economic production quantity models for deteriorating items with price and stock-dependent demand. Comp. Oper. Res. 32, 297-308 (2005) 
26. Dye, C.Y.: The effect of preservation technology investment on a non- instantaneous deteriorating inventory model. Omega 41, 872-880 (2013)

27. Ghiani, Y., Williams, T.: A two- level production inventory model for deteriorating items with multiple buyers. Int. J. Prod. Res. 159, 233-240 (2015)

28. Shah, N.H., Jani, M.Y., Chaudhari, U.: Optimal replenishment time for a retailer under partial upstream prepayment and partial downstream overdue payment for quadratic demand. Math. Comp. Mod. Dyna. Sys. 24(1), 1-11 (2018)

29. Buzacott, J.A.: Economic order quantities with inflation. Oper. Res. Quar. 26, 553-558 (1975)

30. Yang, H.L., Teng, J.T., Chern, M.G.: Deterministic inventory lot- size models under inflation with shortages and deterioration for fluctuating demand. Nov. Res. Log. 48, 144-158 (2001)

31. Sarkar, B., Moon, I.: An EPQ model with inflation in an imperfect production system. App. Math. Comp. 217, 6159-6167 (2011)

32. Chaudhari, U.B., Shah, N.H., Jani, M.Y.: Inventory modelling of deteriorating item and preservation technology with advance payment scheme under quadratic demand. Opt. Inv. Mana. 3(2), 69-79 (2020)

33. Sarkar, B.R., Jamal, A.M.M., Wang, J.: Supply chain model for perishable products under inflation and permissible delay in payments. Comp. Oper. Res. 27, 59-75 (2000)

34. Yang, H.L.: Two warehouse inventory models for deteriorating items with shortages under inflation. Eur. J. Oper. Res. 157, 344-356 (2004)

35. Dey, J.K., Mondal, S.K., Maity, M.: Two storage inventory problem with dynamic demand and interval valued lead time over finite time horizon under inflation and time value of money. Euro. J. Oper. Res. 185, 170-194 (2008)

36. Tripathi, R.P., Chaudhary, S.K.: Inflationary induced EOQ model for Weibull distribution deterioration and trade credits. Int. J. App. Comp. Math. 3(4), 3341-3353 (2017)

37. Tripathi, R.P.: Innovation of Economic Order quantity (EOQ) model for deteriorating items with timelinked quadratic demand under non- decreasing shortages. Int. J. App. Comp. Math. 5(5), 1-13 (2019)

38. Pal, M., Chandra, S.: A periodic review inventory model with stock- dependent demand, permissible delay in payments and price discount in backorders. Yugo. J. Oper. Res. 24(1), 99-110 (2014)

39. Chern, S.C., Cardenss- Barron, L.E., Teng, J.T.: Retailer's economic order quantity when the supplier offers conditionally permissible delay in payments linked to order quantity. Int. J. Pro. Eco. 155, 284-291 (2014)

40. Jiangtao, M., Guimei, C., Ting, F., Hong, M.: Optimal ordering policies for perishable multi- item under stock- dependent demand and two level trade credits. App. Math. Mod. 38(9-10), 2422-2532 (2014)

41. Chen, S.C., Teng, T.: Retailer's optimal ordering policy for deteriorating items with maximum lifetime under supplier's trade credit financing. App. Math. Mod. 38, 4049-4061 (2014)

Publisher's Note Springer Nature remains neutral with regard to jurisdictional claims in published maps and institutional affiliations. 